\title{
LncRNA ZEB1-AS1 knockdown alleviates oxidative low-density lipoprotein-induced endothelial cell injury via the miR-590-5p/ HDAC9 axis
}

\author{
JINPENG ZHONG ${ }^{1 *}$, BIN CHENG ${ }^{2 *}$, LI YANG ${ }^{3}$, GUANLAN LI ${ }^{2}, Y U N Z H O N G ~ Y U A N^{2}$, GANG LUO ${ }^{2}$, \\ ZHIPING SHU ${ }^{2}$, HONG JIANG ${ }^{l}$
}

${ }^{1}$ Department of Cardiology, Renmin Hospital of Wuhan University, Wuhan, Hubei, China

${ }^{2}$ Department of Cardiology, The People's Hospital of China Three Gorges University, Yichang, Hubei, China

${ }^{3}$ Department of Neurology, The People's Hospital of China Three Gorges University, Yichang, Hubei, China

${ }^{\#}$ Jinpeng Zhong and Bin Cheng contributed equally to this work.

\begin{abstract}
Oxidative low-density lipoprotein (ox-LDL) is thought to induce vascular endothelial cell injury, which contributes to the aetiopathogenesis of atherosclerosis (AS). Several previous reports have identified that lncRNA ZEB1-AS1 participates in the regulatory mechanisms of endothelial cell injury, but the potential interaction mechanism between ZEB1-AS1 and miR-590-5p in ox-LDL-induced endothelial cell damage is not clear. ZEB1-AS1 and miR-590-5p expression were tested by quantitative real-time polymerase chain reaction ( $q R T-P C R)$ in ox-LDL-treated endothelial cells. The proliferation and apoptosis were determined by MTT and Annexin V/PI double-staining assay, respectively. The protein expression of HDAC9, tumor necrosis factor $\alpha(T N F-\alpha)$, cleaved caspase-3, and cleaved PARP were measured by western blot analysis. Dual-luciferase reporter and RIP assays affirmed the functional targets of ZEB1AS1. ZEB1-AS1 expression was upregulated in ox-LDL-treated HUVECs, and miR-590-5p was lessened in a dose- or time-depended manner, respectively. Knockdown of ZEB1-AS1 facilitated ox-LDL-treated endothelial cell proliferation and inhibited cell apoptosis. Moreover, miR-590-5p was directly targeted via ZEB1-AS1 in ox-LDL-treated HUVECs. ZEB1-AS1 silencing attenuated ox-LDL-induced cell injury via regulation of miR-590-5p expression. Furthermore, HDAC9 reversed the influence of miR-590-5p on propagation and apoptosis of ox-LDL-induced endothelial cells. Knockdown of ZEB1-AS1 alleviates ox-LDL-induced endothelial cell injury by regulating the miR-590-5p/HDAC9 axis.
\end{abstract}

Key words: IncRNA ZEB1-AS1, miR-590-5p, HDAC9, ox-LDL, atherosclerosis.

(Cent Eur J Immunol 2021; 46 (3): 325-335)

\section{Introduction}

Atherosclerosis (AS) is a complex and chronic inflammatory disease, which is considered as one of the most common vascular diseases $[1,2]$. Oxidative stress is a common mediator in the pathobiology of risk factors for cardiovascular disease [3], and endothelial cell injury caused by oxidative low-density lipoprotein (ox-LDL) is usually characterized as an early marker for AS [4]. Recent reviews have shown that ox-LDL is mainly taken up by macrophage scavenger receptors, which trigger pro-inflammatory cytokine release and further cause endothelial cell dysfunction [5, 6].

Recently, accumulating proof indicates that the long noncoding RNAs (IncRNAs) function as pivotal regulators in diseases [7, 8]. Meanwhile, numerous lncRNAs were identified to be involved in the physiology and tumourigenesis. For example, lncRNA ZEB1-AS1 was identified as a cancerogenic regulator in cancer progression [9]. It is currently considered to be a crucial cancer-related lncRNA. It was observed to be highly expressed in oesophageal squamous cell carcinoma [10], osteosarcoma [11], bladder cancer [12], glioma [13], colorectal cancer [14], and B-lymphoblastic leukaemia [15]. Li et al. summarized current evidence concerning the ZEB1-AS1 in the form of tables. The combined data show that ZEB1-AS1 acts as either a tumour inhibitor or as a carcinogen during tumourigenesis. However, the function and regulatory mechanism underlying ZEB1-AS1 in ox-LDL-induced endothelial cells still need further investigation. 
In addition, non-coding RNAs, including lncRNA and microRNAs (miRNAs), play crucial roles in the occurrence and progression of cancer by regulating several physiological and pathological processes [16]. LncRNA are longer than 200 nucleotides in length, while miRNA as endogenous small RNA consists of 18-24 nucleotides. LncRNA acts as a competitive endogenous RNA to interact with miRNA [17, $18]$. Both of these play a crucial regulatory role in the complex mechanisms of cancer development [19].

In a previous study, ZEB1-AS1 played a role in oxLDL-induced endothelial cell damage [20]. On the basis of the above findings, in order to explore ZEB1-AS1/ miR-590-5p underlying the molecular relationship in oxLDL-treated endothelial cell damage, the effects of the ZEB1-AS1/miR-590-5p/HDAC9 axis on ox-LDL-mediated endothelial cell proliferation and apoptosis were investigated.

\section{Material and methods}

\section{Samples and ethics statement}

Blood samples were obtained from 30 normal persons and 30 AS patients in Renmin Hospital of Wuhan University. Serum was obtained from the blood after standing overnight at $4^{\circ} \mathrm{C}$, and then stored at $-20^{\circ} \mathrm{C}$ until total RNAs were extracted. This protocol was authorised by the Ethical Committee of Renmin Hospital of Wuhan University, and all patients gave written informed consent.

\section{Cell culture and ox-LDL treatment}

Human umbilical vein endothelial cells (HUVECs) were obtained from the Bena Culture Collection (Suzhou, Jiangsu, China). At $37^{\circ} \mathrm{C}$ and $5 \% \mathrm{CO}_{2}$, HUVECs were grown in RPMI 1640 medium (Gibco, Grand Island, NY, USA) containing $10 \%$ foetal bovine serum (FBS, Gibco) and $1 \%$ antibiotics (Gibco). Ox-LDL (UnionBiol, Beijing, China) was purchased and diluted to different concentration gradients. HUVECs were incubated with increasing concentrations of ox-LDL $(0,25,50,100 \mu \mathrm{g} / \mathrm{ml})$ for $24 \mathrm{~h}$ or at different points in time $(0 \mathrm{~h}, 12 \mathrm{~h}, 24 \mathrm{~h}, 48 \mathrm{~h})$ at the concentration of $50 \mu \mathrm{g} / \mathrm{ml}$, and then a quantitative real-time polymerase chain reaction (qRT-PCR) assay was utilized to estimate the level of ZEB1-AS1 and miR-590-5p in HUVECs.

\section{qRT-PCR reaction}

Total RNA from serum samples or cells was extracted using TRIzol (Takara, Dalian, China) and transcribed into cDNA via FastQuant RT reagent (Takara), and qRTPCR was conducted with SYBR Green I Kit (Takara) on an ABI 7500 HT system (Applied Biosystems, Carlsbad, CA, USA). All data were normalized to U6 or GAPDH. The relative expression of genes was calculated using the $2^{-\Delta \Delta \mathrm{Ct}}$ method [21]. The main primer sequences for qRT-PCR were as follows: ZEB1-AS1, forward, ATTT-
GAATTGAGGGGCGAGG and reverse, GGAAACCAGGCGTCCCTTT; U6, forward, CTCGCTTCGGCAGCACA and reverse, AACGCTTCACGAATTTG; HDAC9, forward, AGTAGAGAGGCATCGCAGAGA and reverse, GGAGTGTCTTTCGTTGCTGAT; GAPDH, forward, GCACCGTCAAGGCTGAGAAC and reverse, ATGGTGGTGAAGACGCCAGT. Besides, Silencing RNA sequences were as follows: si-ZEB1-AS1\#1, GACCGGAGUAUUGGAAAUA; si-ZEB1-AS1\#2, GGGCACUGCUGAAUUUGAA; si-ZEB1-AS1\#3, GCUGAAGUCUGAUGAUUUA; si-NC, GACCTACAACTACCTATCA.

\section{Cell transfection}

Interference fragment of ZEB1-AS1 (si-ZEB1-AS1\#1, si-ZEB1-AS1\#2, si-ZEB1-AS1\#3), miR-590-5p inhibitor (anti-miR-590-5p), overexpression of HDAC9 (pcDNAHDAC9), and their negative controls (si-NC, anti-miRNC, or pcDNA) were received from RiboBio (Guangdong, China). HUVECs were transiently transfected using Lipofectamine 3000 reagent (Invitrogen, Carlsbad, CA, USA) and induced by ox-LDL $(100 \mu \mathrm{g} / \mathrm{ml})$ for the later experiments.

\section{MTT assay}

HUVECs were grown after ox-LDL induction in a 96-well flat-bottomed plate. MTT solution was added into cell culture wells and incubated with cells for $4 \mathrm{~h}$ at a temperature of $37^{\circ} \mathrm{C}$. The upper media was taken away, and then DMSO was utilized to dissolve formazan crystals. Finally, the absorbance of cells was determined at $490 \mathrm{~nm}$. All assays were conducted more than 2 times.

\section{Annexin V/PI double-staining assay}

HUVECs were grown and exposed with the indicated concentrations of ox-LDL. After a certain period of time, cells were harvested and then stained with annexin V-FITC and PI. The apoptosis rates of cells were evaluated by flow cytometry (Nikon, Tokyo, Japan) and analysed using software. Each assay was performed 3 times.

\section{Western blot assay}

Well-grown cells were treated with ox-LDL. Total protein extraction was performed via RIPA buffer (Pierce, Rockford, USA). Protein was segregated by SDS-PAGE after quantification, and then transferred onto a PVDF membrane (Millipore, Billerica, MA, USA). The blots were incubated with primary antibodies against tumor necrosis factor $\alpha$ (TNF- $\alpha)(1: 200$, Abcam, Cambridge, United Kingdom), cleaved caspase-3 (1:1000, Abcam), cleaved PARP (1:1000, Abcam), or GAPDH (1:2000, Abcam, UK), and then added with secondary antibody (1: 5000, Abcam). Finally, the signal was detected via an ECL Plus Detection kit (Pierce). 


\section{Dual-luciferase reporter assay}

The binding sites of ZEB1-AS1 and miR-590-5p were predicted by StarBase v2.0. The potential target HDAC9 of miR-590-5p was searched with TargetScan. The wild-type or mutant ZEB1-AS1 (ZEB1-AS1-wt and ZEB1-AS1-mut) and HDAC9-3'UTR (HDAC9wt and HDAC9-mut) fragments were constructed and inserted into pMIRGLO ${ }^{\text {TM }}$ luciferase vectors (Promega, Madison, WI, USA). Ox-LDL-treated HUVECs were cotransfected with above plasmids and miR-590$5 \mathrm{p}$ or miR-NC by using Lipofectamine 3000 (Invitrogen). Then, luciferase activity was detected via DualLuciferase Assay Kit (Promega).

\section{RNA immunoprecipitation (RIP) assay}

To attest the relationship between ZEB1-AS1 and miR-590-5p, an RIP assay by EZ-Magna RIP kit (Millipore) was used. Briefly, ox-LDL-treated HUVECs were obtained and resolved in RIP lysis buffer. Next, the cell lysates were incubated with protein $\mathrm{A} / \mathrm{G}$ bead-conjugated anti-Argonaute2 (Ago2) antibody (Abcam) or control immunoglobulin $\mathrm{G}$ (IgG) antibody (Abcam) at $4{ }^{\circ} \mathrm{C}$ for $4 \mathrm{~h}$. Finally, co-precipitated RNA was measured by qRT-PCR analysis.

\section{Statistical analysis}

All data were obtained from at least 3 replications and presented as the mean \pm standard deviation (SD). Analysis of the differences between groups was conducted via Student's $t$-test or one-way ANOVA. $P<0.05$ was deemed to imply statistical significance.

\section{Results}

\section{ZEB1-AS1 was upregulated in AS patient serums and ox-LDL-treated HUVECs, the opposite of miR-590-5p}

In order to explore the relationship between genes expression levels in the serums of normal persons and patients the levels of ZEB1-AS1 and miR-590-5p and ox-LDL treatment dose or treatment time were studied. The ZEB1AS1 level was increased in AS patient serums relative to that of normal persons (Fig. 1A), which was accompanied by dose-dependent ox-LDL-induced endothelial cell injury. The expression level of ZEB1-AS1 was the highest at $100 \mu \mathrm{g} / \mathrm{ml}$, compared to other concentrations (Fig. 1B). Similarly, ZEB1-AS1 was upregulated in a time-dependent fashion after ox-LDL-challenged injury of endothelial cells at the concentration of $100 \mu \mathrm{g} / \mathrm{ml}$ (Fig. 1C). Moreover, a reduced miR-590-5p level was shown in AS patients (Fig. 1D), and miR-590-5p abundance was lowered followed by the addition of incremental concentration of ox-LDL treatment in HUVECs (Fig. 1E), as well as the
ox-LDL treatment time in HUVECs (Fig. 1F), which was exactly the opposite of the expression trend of ZEB1-AS1. The results indicated that the ox-LDL concentration of $100 \mu \mathrm{g} / \mathrm{ml}$ should be used for further experiments. Hence, HUVECs that treated with $100 \mu \mathrm{g} / \mathrm{ml}$ ox-LDL for $24 \mathrm{~h}$ were employed as an in vitro cellular model for AS in subsequent research.

\section{Knockdown of ZEB1-AS1 promoted ox-LDL-treated HUVEC proliferation and inhibited HUVEC apoptosis}

To explore the potential character of ZEB1-AS1 in oxLDL-induced HUVEC injury, the HUVECs were treated with ox-LDL for $24 \mathrm{~h}$ at $100 \mu \mathrm{g} / \mathrm{ml}$. ZEB1-AS1 was silenced by using 3 specific silencing oligonucleotides (siZEB1-AS1\#1, si-ZEB1-AS1\#2, and si-ZEB1-AS1\#3) in HUVECs. The qRT-PCR results demonstrated that the abundance of ZEB1-AS1 was significantly downregulated compared with negative controls after si-ZEB1-AS1\#1 transfection (Fig. 2A); thereby, it was utilized for subsequent study. MTT assay indicated that ZEB1-AS1 silence promoted ox-LDL-treated endothelial cell proliferation (Fig. 2B). ZEB1-AS1 interference overtly overturned the accelerated effect of ox-LDL addition on LDH liberation (Fig. 2C). Flow cytometry results showed that knockdown of ZEB1-AS1 inhibited HUVEC apoptosis (Fig. 2D). In addition, western blot assay showed that the levels of TNF- $\alpha$, cleaved caspase- 3 , and cleaved PARP were significantly downregulated after ZEB1-AS1 silencing (Fig. 2E). These data suggest that knockdown of ZEB1-AS1 facilitated the proliferation of endothelial cells induced by ox-LDL and inhibited endothelial cell apoptosis in vitro.

\section{MiR-590-5p was targeted by ZEB1-AS1 in ox-LDL-treated HUVECs}

Considering the opposite expression of ZEB1-AS1 and miR-590-5p in the ox-LDL-treated HUVECs, we speculated that ZEB1-AS1 and miR-590-5p may have a targeted relationship. As expected, Starbase software showed that ZEB1-AS1 had complementary sequence with miR-590-5p (Fig. 3A). Luciferase reporter results showed that miR590-5p visibly lessened the luciferase expression of ZEB1AS1-WT in ox-LDL-treated HUVECs but did not affect that of ZEB1-AS1-MUT (Fig. 3B). RIP assay suggested that ZEB1-AS1 and miR-590-5p were both particularly enriched in HUVEC extracts of Ago2 group compared to that of the IgG group (Fig. 3C). The level of miR-590-5p was distinctly raised after ZEB1-AS1 knockdown, and ZEB1-AS1 overexpression inhibited miR-590-5p expression levels (Fig. 3D). The abundance of ZEB1-AS1 and miR-590-5p had a significant negative correlation (Fig. 3E). Collectively, these findings hinted that ZEB1-AS1 could directly bind to miR-590-5p and served as a competing endogenous RNA (ceRNA). 

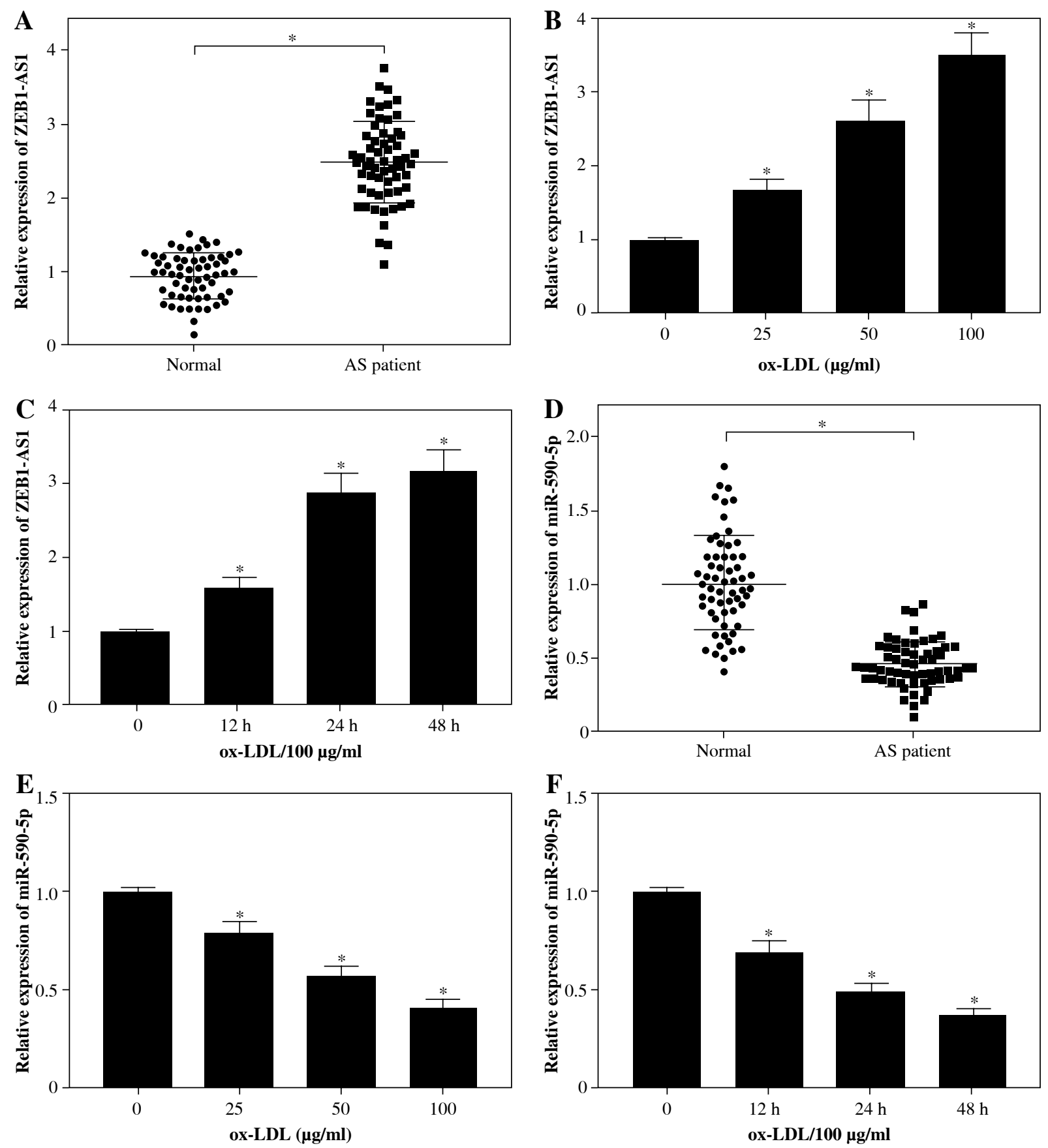

Fig. 1. Expression of ZEB1-AS1 and miR-590-5p in the serum of AS patients and ox-LDL-treated HUVECs. A, D) The levels of ZEB1-AS1 and miR-590-5p were detected by qRT-PCR in AS patient serums. B) HUVECs were treated with $0,25,50$, and $100 \mu \mathrm{g} / \mathrm{ml}$ ox-LDL for $24 \mathrm{~h}$. C) HUVECs were treated with $100 \mu \mathrm{g} / \mathrm{ml}$ ox-LDL for $0,12,24$, and 48 h. E, F) Ox-LDL treatment decreased miR-590-5p expression in a dose- or time-dependent manner. $* p<0.05$

ZEB1-AS1 silencing attenuated ox-LDL-induced endothelial cell injury through regulation of miR-590-5p

Inhibition of ZEB1-AS1 could significantly increase miR-590-5p expression, while inhibition of miR-590-5p could significantly mitigate the effect of the ZEB1-AS1 knockdown (Fig. 4A). MTT assay showed that ZEB1-AS1 silencing promoted ox-LDL-induced endothelial cell proliferation (Fig. 4B) and decreased the release of LDH. While ZEB1-AS1 was knocked down, miR-590-5p silencing could abolish these effects (Fig. 4C). ZEB1-AS1 silencing attenu- 

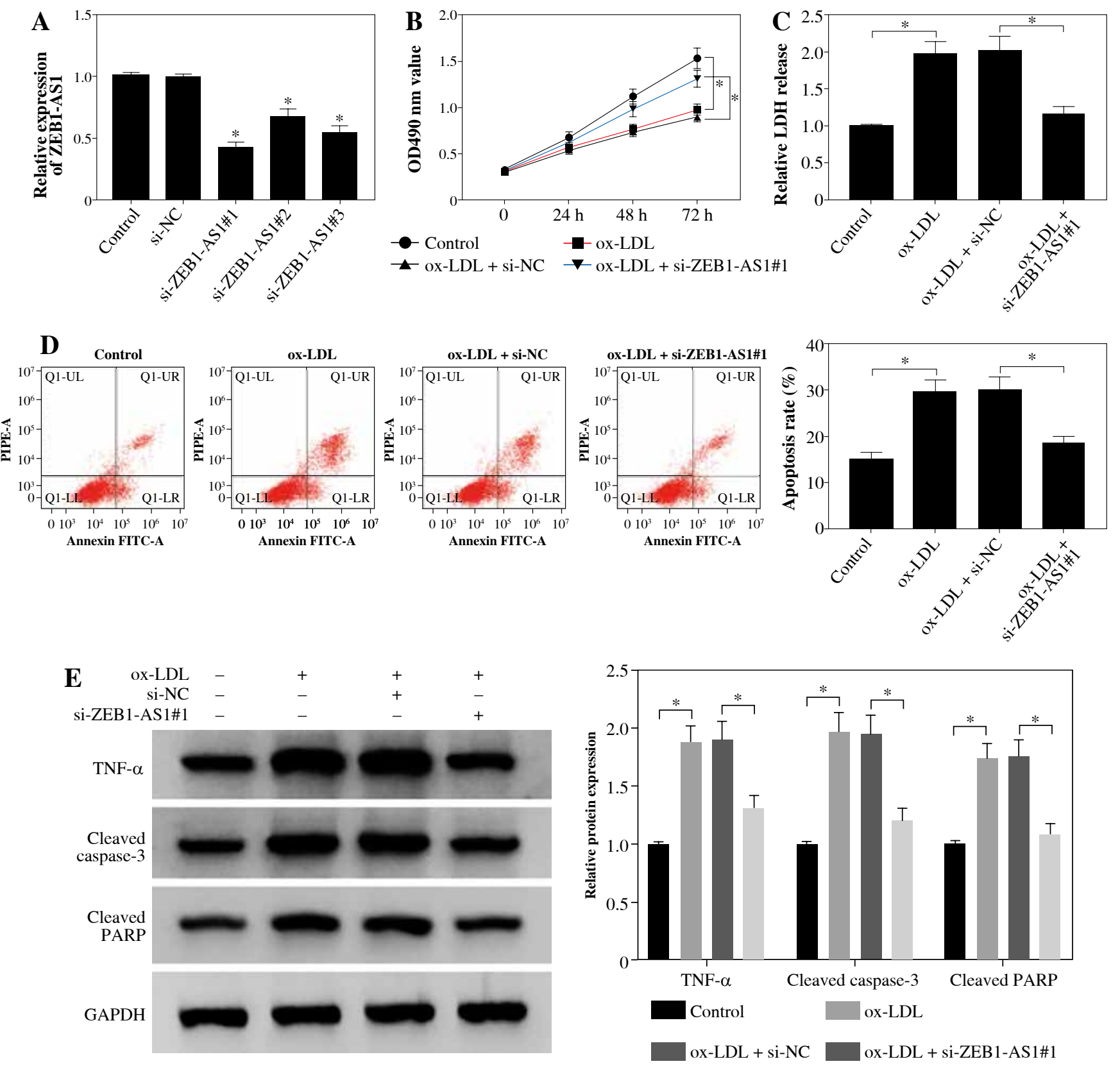

Fig. 2. ZEB1-AS1 silencing promoted ox-LDL-treated endothelial cell proliferation and inhibited endothelial cell apoptosis. A) ZEB1-AS1 was silenced by transfection of specific ZEB1-AS1 siRNAs (si-ZEB1-AS1\#1, si-ZEB1-AS1\#2, and si-ZEB1-AS1\#3). B, C) HUVECs (treated with ox-LDL or not) were transfected with si-NC or si-ZEB1-AS1\#1. Cell viability $(\mathbf{B})$ and LDH release $(\mathbf{C})$ were measured by MTT assay and LDH Cytotoxicity Assay Kit, respectively. D) HUVEC apoptosis was detected after treatment with ox-LDL or knockdown of ZEB1-AS1 for 24 h. E) The protein levels of TNF- $\alpha$, cleaved caspase-3, and cleaved PARP were measured in ox-LDL-induced HUVECs transfected with si-ZEB1-AS1\#1. * $p<0.05$

ated ox-LDL-induced apoptosis of endothelial cells through regulation of miR-590-5p (Fig. 4D). In addition, knockdown of ZEB1-AS1 inhibited TNF- $\alpha$, cleaved caspase-3, and cleaved PARP protein levels (Fig. 4E). The data indicate that ZEB1-AS1 silencing attenuated ox-LDL-induced endothelial cell damage via regulation of miR-590-5p.

\section{ZEB1-AS1 regulated HDAC9 expression via miR-590-5p}

TargetScan suggested that 3'UTR of HDAC9 contained with the miR-590-5p seed sites, and the bases of the binding site sequence were mutated (Fig. 5A). To eval- 

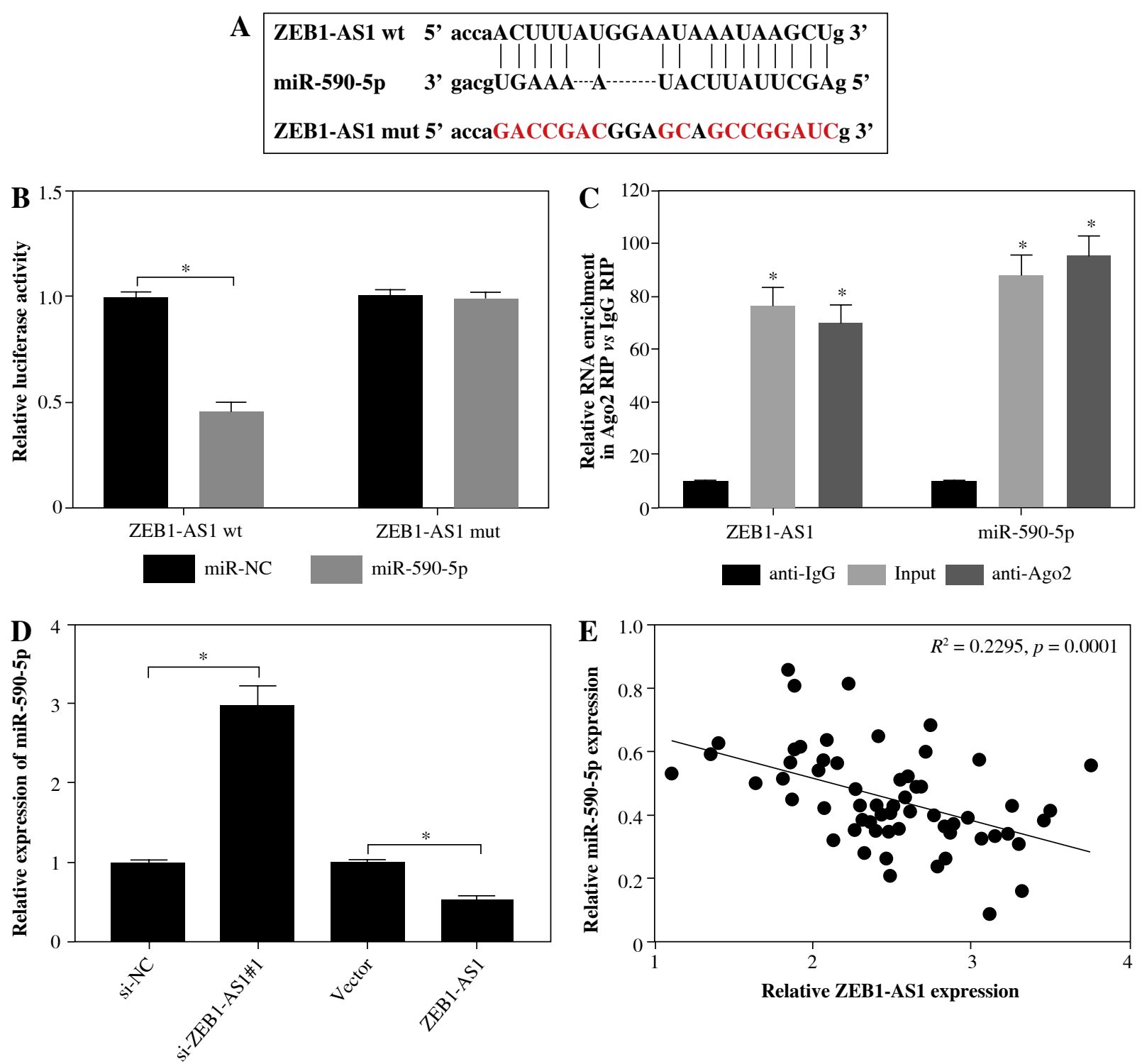

Fig. 3. miR-590-5p is a direct target of ZEB1-AS1 in ox-LDL-treated HUVECs. A) The predicted binding sites of miR590-5p in ZEB1-AS1. B) Luciferase activity was detected in ox-LDL-treated HUVECs cotransfected with luciferase vectors containing ZEB1-AS1-wt or ZEB1-AS1-mut and miR-NC or miR-590-5p. C) RIP assay was conducted in oxLDL-treated HUVECs extracts to examine miR-590-5p endogenously associated with ZEB1-AS1. D) The expression of miR-590-5p in ox-LDL-treated HUVECs transfected with si-NC, si-ZEB1-AS1\#1, Vector or ZEB1-AS1 was detected by qRT-PCR assay. E) The negative correlation between ZEB1-AS1 and miR-590-5p in ox-LDL-treated HUVECs $\left(R^{2}=0.2295, p=0.0001\right) . * p<0.05$

uate the direct binding between miR-590-5p and HDAC9, dual-luciferase reporter assay was implemented. It was certified that miR-590-5p observably decreased the luciferase activity of HDAC9-wt plasmid $(p<0.05)$ but had no effect on that of HDAC9-mut plasmid (Fig. 5B). Next, qRT-PCR and western blot assay showed that HDAC9 was strongly downregulated after miR-590-5p transfection in HUVECs and was upregulated after miR-590-5p interference in mRNA and protein levels (Fig. 5C, D). MiR-590$5 p$ inhibition partially reversed the effects of ZEB1-AS1 knockdown on HDAC9 expression of ox-LDL-treated
HUVECs (Fig. 5E), as well as HDAC9 protein expression levels (Fig. 5F). All data indicate that ZEB1-AS1 regulated HDAC9 expression by targeting miR-590-5p.

\section{HDAC9 reversed the influence of miR-590-5p on proliferation and apoptosis of ox-LDL-induced endothelial cells}

To explore the functional relationship between miR590-5p and HDAC9 in ox-LDL-induced endothelial cells, the expression of HDAC9 was measured in HUVECs after 

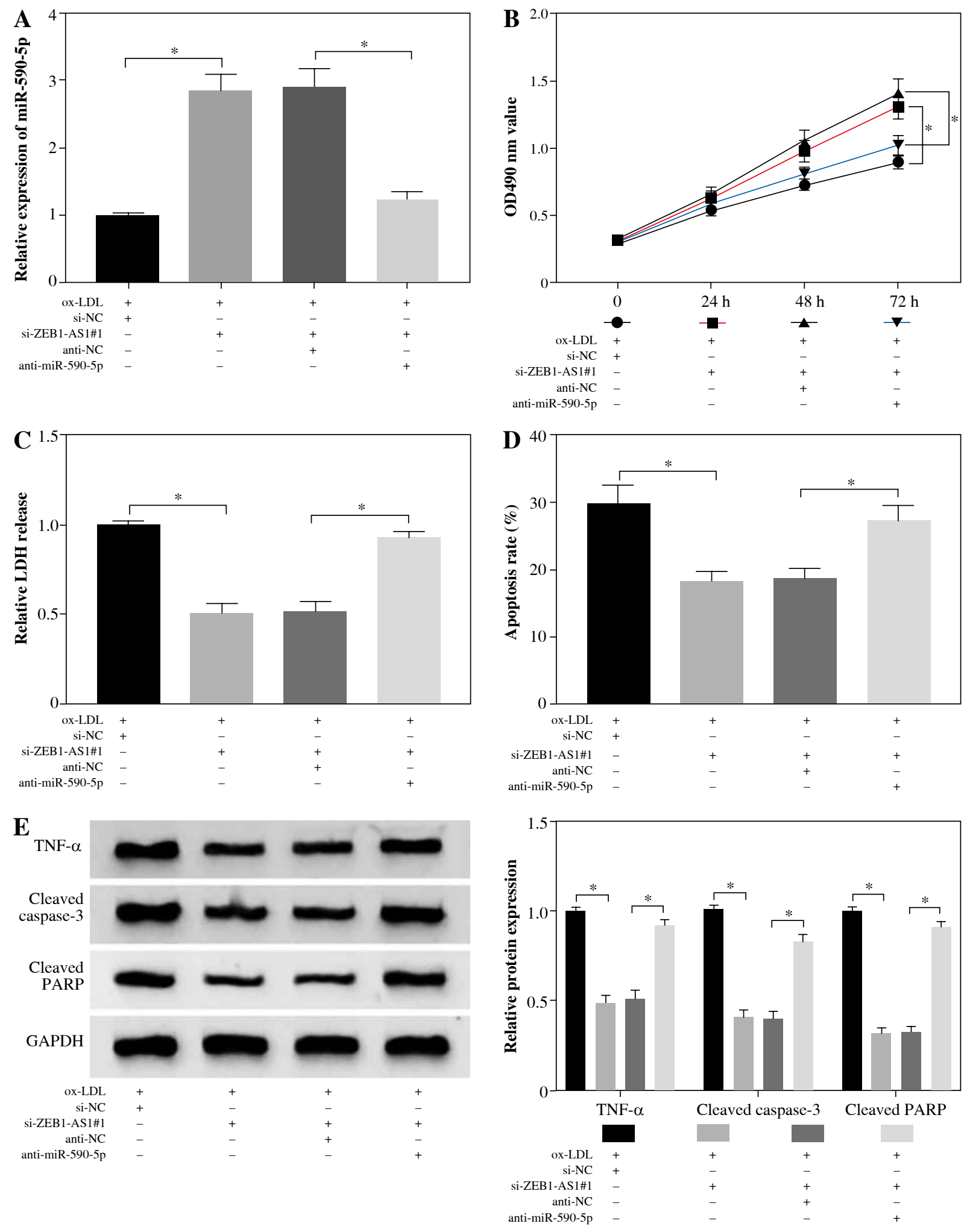

Fig. 4. ZEB1-AS1 silencing attenuated ox-LDL-induced endothelial cell injury through regulation of miR-590-5p. After treatment with ox-LDL, the HUVECs were transfected with si-NC, si-ZEB1-AS1\#1, anti-NC, or anti-miR-590-5p + siZEB1-AS1\#1. A) The expression levels of miR-590-5p were detected by qRT-PCR. B) The viability of ox-LDL-induced HUVECs was detected at the indicated time points by MTT. C) Relative LDH release of ox-LDL-induced HUVECs was analysed by ELISA. D) The apoptosis of HUVECs were detected by using flow cytometry. E) The TNF- $\alpha$, cleaved caspase-3, and cleaved PARP protein expressions were measured by western blot analysis. $* p<0.05$ 
A

\begin{tabular}{|lr|}
\hline HDAC9 wt 3'UTR & 5'...uagguuuACUUUUgu--.--UAAGCUag...3' \\
miR-590-5p & 3' gacgUGAAAAuacuuAUUCGAg \\
HDAC9 mut 3'UTR & 5 '...uagguuuGACGCGgu--.--GCGAUCag....3' \\
\hline
\end{tabular}

B
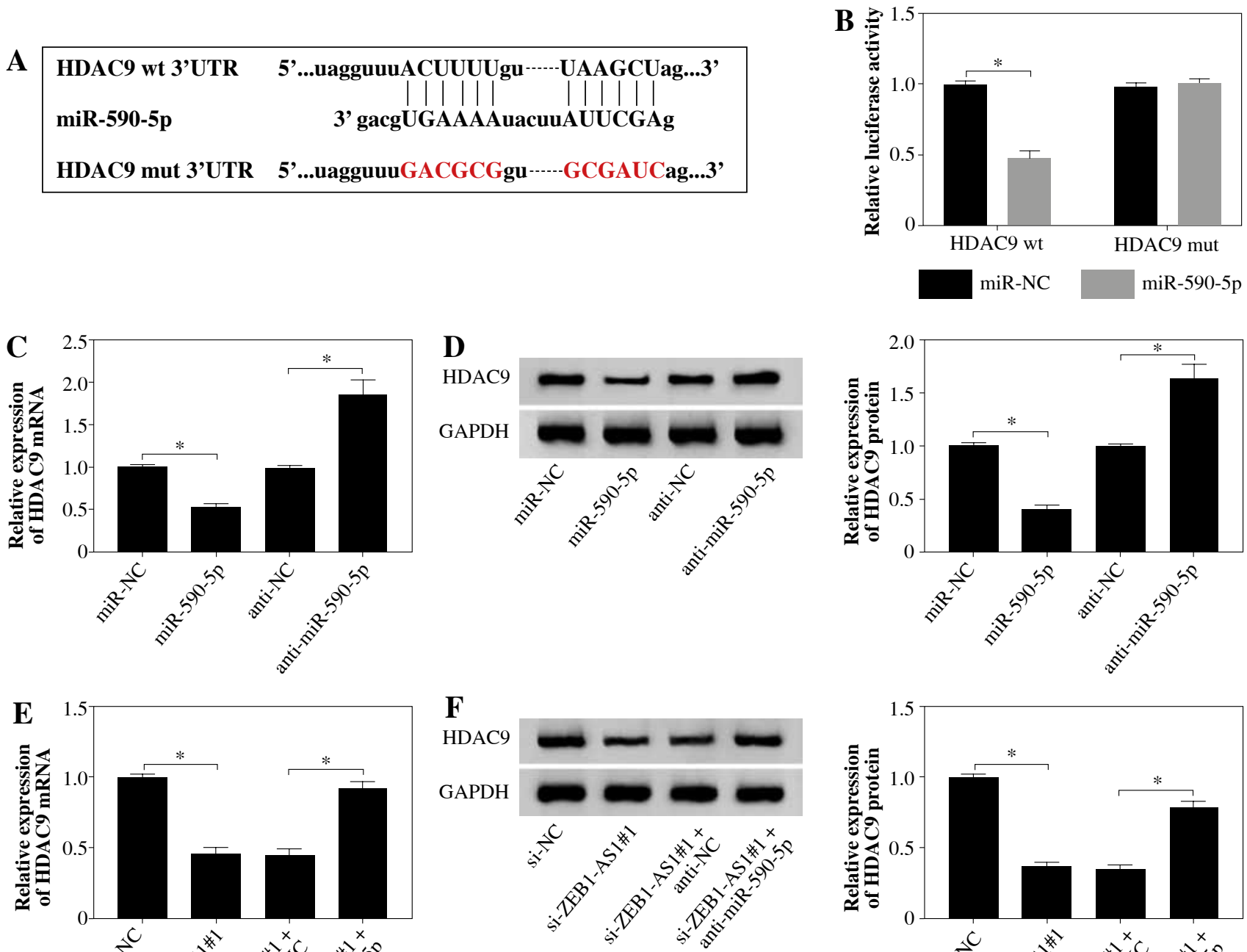

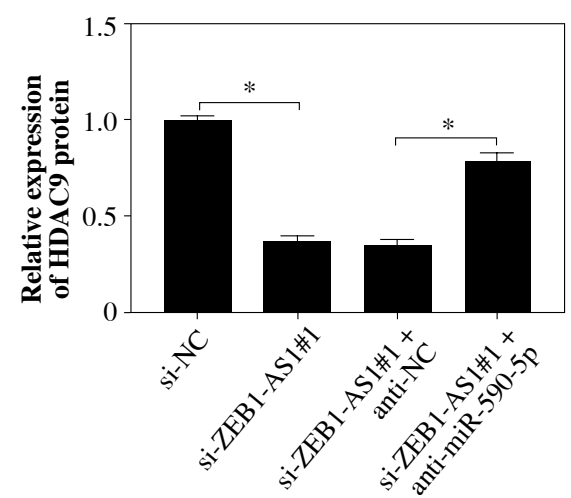

Fig. 5. ZEB1-AS1 targeted miR-590-5p to regulate HDAC9 expression. A) The predicted binding sites of miR-590-5p in 3'-UTR of HDAC9. B) The wild-type or mutated HDAC9 3'-UTR reporter plasmids were co-transfected into ox-LDLinduced HUVECs with miR-NC or miR-590-5p, and then luciferase activity was detected. C, D) Expression levels of HDAC9 mRNA and protein were examined by qRT-PCR $(\mathbf{C})$ and western blot $(\mathbf{D})$ in ox-LDL-induced HUVECs treated with miR-NC, miR-590-5p, anti-NC, or anti-miR-590-5p, respectively. E, F) Expression levels of HDAC9 mRNA and protein were examined by qRT-PCR $(\mathbf{E})$ and western blot $(\mathbf{F})$ in ox-LDL-induced HUVECs treated with si-NC, si-ZEB1AS1\#1, si-ZEB1-AS1\#1 + anti-NC, and si-ZEB1-AS1\#1 + anti-miR-590-5p, respectively. * $p<0.05$

miR-590-5p overexpression and pcDNA-HDAC9, respectively. As presented in Figure 6A, B, miR-590-5p overexpression dramatically repressed expression of HDAC9 in mRNA and protein levels; however, this effect was evidently improved by HDAC 9 overexpression. Furthermore, HUVECs were cotransfected with miR-590-5p mimics and pcDNA-HDAC9 to investigate their roles in cell progression. MTT assay demonstrated that overexpression of miR590-5p overtly enhanced ox-LDL-induced HUVEC proliferation (Fig. 6C). Relative LDH release was decreased in
HUVECs after overexpression of miR-590-5p (Fig. 6D), whereas HDAC9 supplement relieved these effects. Meanwhile, the apoptosis of HUVECs transfected with pcDNAHDAC9 vector was dramatically increased compared with control groups (Fig. 6E). In addition, the introduction of miR-590-5p triggered an evident reduction in TNF- $\alpha$, cleaved caspase-3, and cleaved PARP expression in oxLDL-induced HUVECs, and pcDNA-HDAC9 reversed the above effects (Fig. 6F). These data suggest that HDAC9 reversed the impact of miR-590-5p on proliferation and apoptosis of ox-LDL-induced endothelial cells. 

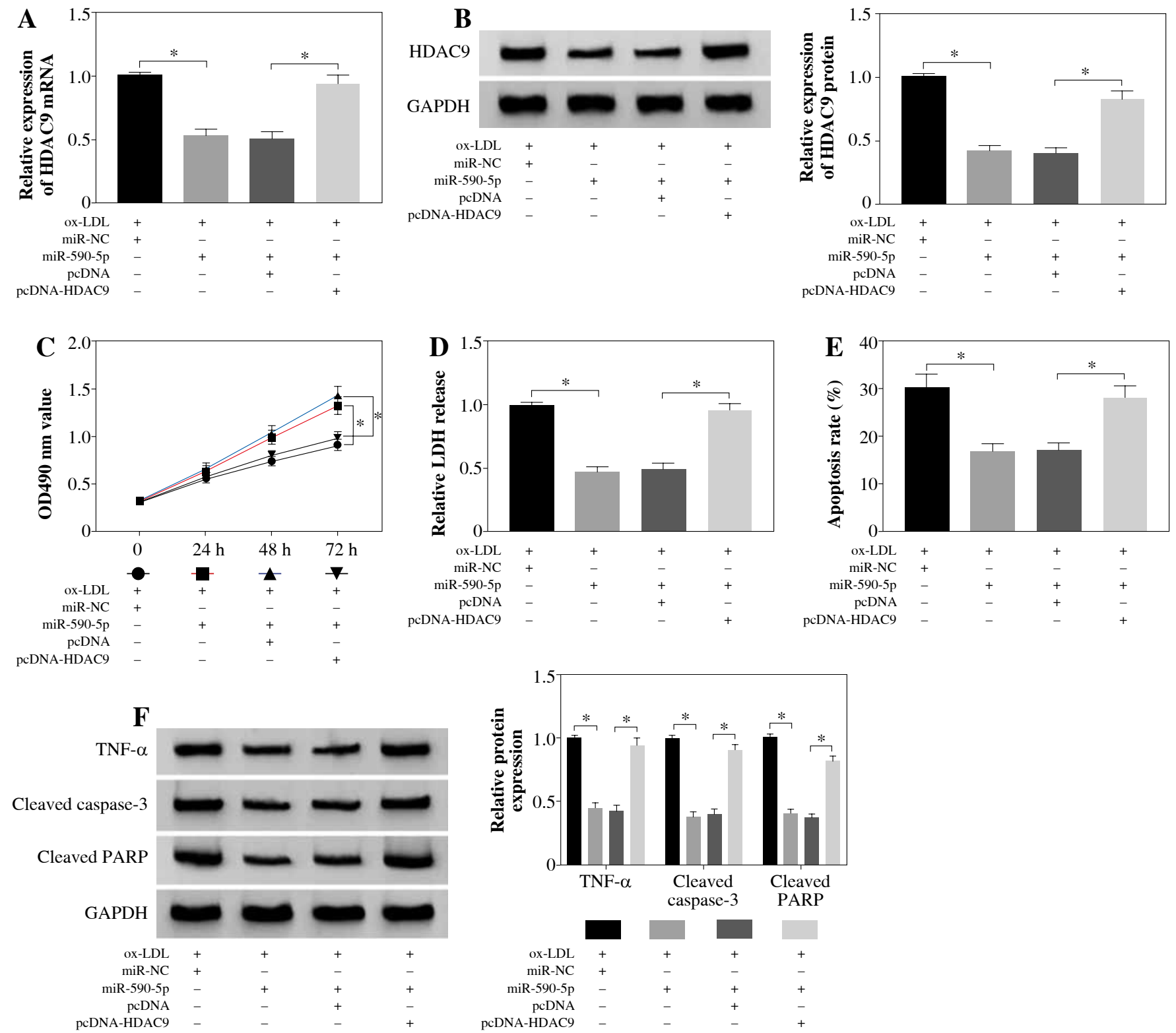

Fig. 6. HDAC9 reversed the effect of miR-590-5p on proliferation and apoptosis of ox-LDL-induced endothelial cells. After treatment with ox-LDL, the HUVECs were transfected with miR-NC, miR-590-5p, pcDNA, or pcDNA-HDAC9. A, B) The expression levels of HDAC9 mRNA and protein were detected by qRT-PCR and western blot analysis, respectively. C, D) Cell viability and LDH release were measured by MTT assay and ELISA, respectively. E) HUVEC apoptosis was detected by using flow cytometry assay. F) The TNF- $\alpha$, cleaved caspase- 3 , and cleaved PARP protein expression were measured by western blot analysis. $* p<0.05$

\section{Discussion}

Recently, the causal mechanisms of AS development have been explored by more and more researchers. According to previous studies, lipid metabolism disorder is the basis of AS lesions. Moreover, ox-LDL-mediated endothelial cell injury is a crucial factor in the development and progression of AS [22, 23]. AS is an inflammatory disease $[24,25]$. It is the primary cause of coronary heart disease, cerebral infarction, and peripheral vascular disease, which are among the leading causes of disease death in the world today [26]. Therefore, it is highly important to inspect the mechanism of ox-LDL-induced endothelial cell injury.

Emerging studies suggest a crucial regulatory effect of lncRNAs in human diseases, particularly in tumouri- 
genesis. For example, the lncRNA MALAT1 level was significantly higher in patients with unstable angina and could protect the endothelium from ox-LDL-induced endothelial dysfunction [27]. LncRNA CCAT1 was also up-regulated in colorectal cancer and was considered as a detectable marker for early detection of colorectal cancer [28]. Studies have shown that lncRNA XIST is upregulated in ox-LDL-mediated HUVECs, and knockdown of XIST alleviates ox-LDL-mediated endothelial cell injury [29]. Therefore, it is necessary to exploit more lncRNAs and target genes and illuminate their underlying mechanism in the development of cancers.

ZEB1-AS1 has been identified as an oncogenic regulator during the occurrence and development of diverse malignancies. Originally, differentially expressed ZEB1-AS1 was identified and investigated in hepatocellular carcinoma [30]. Meanwhile, different splicing variants of ZEB1-AS1 were explained in different cell lines [31]. More importantly, ZEB1-AS1 as a transcription factor functions in the development of cancers [32]. Recently, more and more attention has been paid to the functional roles of ZEB1-AS1 in ox-LDL-mediated endothelial cell injury.

Significant roles of miRNA in AS have been studied. miR-590-5p has been shown to keep endothelial cells from ox-LDL-induced apoptosis by regulating LOX-1 expression [33]. Based on animal models of AS, miR-590 suppressed ox-LDL-induced apoptosis in HAECs through

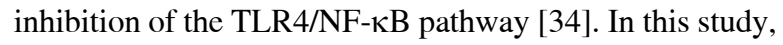
ZEB1-AS1 was increased in ox-LDL-treated HUVECs, the opposite of miR-590-5p, and relative expression of ZEB1AS1 and miR-590-5p had a negative correlation. Further studies indicated that ZEB1-AS1 knockdown enhanced ox-LDL-treated endothelial cell proliferation and inhibited endothelial cell apoptosis, and attenuated ox-LDL-treated endothelial cell injury through regulation of miR-590-5p. Bioinformatics software predicted that miR-590-5p and HDAC9 have targeted binding sites. Previous studies showed that HDAC9 played regulatory roles in ox-LDLinduced endothelial cell inflammation [35]. HDAC9 was differently up-regulated in endothelial cells [36]. The deletion of HDAC9 could reduce the occurrence of inflammation and AS [37]. Previous reviews indicated further roles of HDAC9 in AS and inflammation [38]. Therefore, we speculated the roles of HDAC9 may be involved in endothelial cell damage. In agreement with previous findings, the results in this study showed that HDAC9 reversed the effect of miR-590-5p on proliferation and apoptosis of oxLDL-induced endothelial cells.

This study showed that ZEB1-AS1 regulated HDAC9 expression by affecting miR-590-5p expression in ox-LDLinduced HUVECs. However, a deeper inquiry of whether ZEB1-AS1/miR-590-5p/HDAC9 signalling affects the activation of certain pathways associated with endothelial cell injury will be a subject of our further research.

\section{Conclusions}

We identified the targeting correlation between lncRNA ZEB1-AS1 and miR-590-5p by establishing a model of ox-LDL-induced endothelial cell injury. Moreover, our results further attested that ZEB1-AS1 silencing alleviates ox-LDL-induced endothelial cell damage by regulating the miR-590-5p/HDAC9 signalling axis, which might be a novel strategy to target therapy of endothelial cell injury in AS.

\section{The authors declare no conflict of interest.}

\section{References}

1. Ross R (1999): Atherosclerosis - an inflammatory disease. N Engl J Med 340: 115-126.

2. Hansson GK, Libby P, Schönbeck U, et al. (2002): Innate and adaptive immunity in the pathogenesis of atherosclerosis. Circ Res 91: 281-291.

3. Ho E, Karimi Galougahi K, Liu CC, et al. (2013): Biological markers of oxidative stress: applications to cardiovascular research and practice. Redox Biol 1: 483-491.

4. Chen M, Masaki T, Sawamura T (2002): LOX-1, the receptor for oxidized low-density lipoprotein identified from endothelial cells: implications in endothelial dysfunction and atherosclerosis. Pharmacol Ther 95: 89-100.

5. Pirillo A, Norata GD, Catapano AL (2013): LOX-1, OxLDL, and atherosclerosis. Mediators Inflamm 2013: 152786.

6. Sawamura T, Kume N, Aoyama T, et al. (1997): An endothelial receptor for oxidized low-density lipoprotein. Nature 386: 73-77.

7. Cech TR, Steitz JA (2014): The noncoding RNA revolution-trashing old rules to forge new ones. Cell 157: 77-94.

8. Chen G, Wang Z, Wang D, et al. (2013): LncRNADisease: a database for long-non-coding RNA-associated diseases. Nucleic Acids Res 41: D983-986.

9. Li J, Li Z, Leng K, et al. (2018): ZEB1-AS1: A crucial cancer-related long non-coding RNA. Cell Prolif 51: e12423.

10. Wang YL, Bai Y, Yao WJ, et al. (2015): Expression of long non-coding RNA ZEB1-AS1 in esophageal squamous cell carcinoma and its correlation with tumor progression and patient survival. Int J Clin Exp Pathol 8: 11871-11876.

11. Liu C, Lin J (2016): Long noncoding RNA ZEB1-AS1 acts as an oncogene in osteosarcoma by epigenetically activating ZEB1. Am J Transl Res 8: 4095-4105.

12. Lin J, Zhan Y, Liu Y, et al. (2017): Increased expression of ZEB1-AS1 correlates with higher histopathological grade and promotes tumorigenesis in bladder cancer. Oncotarget 8 : 24202-24212.

13. Lv QL, Hu L, Chen SH, et al. (2016): A long noncoding RNA ZEB1-AS1 promotes tumorigenesis and predicts poor prognosis in glioma. Int J Mol Sci 17: 1431.

14. Gong H, Wen H, Zhu X, et al. (2017): High expression of long non-coding RNA ZEB1-AS1 promotes colorectal cancer cell proliferation partially by suppressing p 15 expression. Tumour Biol 39: 1010428317705336.

15. Wang Q, Du X, Yang M, et al. (2017): LncRNA ZEB1-AS1 contributes to STAT3 activation by associating with IL-11 in B-lymphoblastic leukemia. Biotechnol Lett 39: 1801-1810. 
16. Bartel DP (2004): MicroRNAs: genomics, biogenesis, mechanism, and function. Cell 116: 281-297.

17. Jalali S, Bhartiya D, Lalwani MK, et al. (2013): Systematic transcriptome wide analysis of IncRNA-miRNA interactions. PLoS One 8: e53823.

18. Tay Y, Rinn J, Pandolfi PP (2014): The multilayered complexity of ceRNA crosstalk and competition. Nature 505: 344-352.

19. Ma MZ, Chu BF, Zhang Y, et al. (2015): Long non-coding RNA CCAT1 promotes gallbladder cancer development via negative modulation of miRNA-218-5p. Cell Death Dis 6: e1583.

20. Xu X, Ma C, Duan Z, et al. (2019): IncRNA ZEB1-AS1 mediates oxidative low-density lipoprotein-mediated endothelial cells injury by post-transcriptional stabilization of NOD2. Front Pharmacol 10: 397.

21. Kinoshita T, Imamura J, Nagai H, et al. (1992): Quantification of gene expression over a wide range by the polymerase chain reaction. Anal Biochem 206: 231-235.

22. Sumiyoshi A, Asada Y (1988): Endothelial injury as an initiating factor: mechanism and significance of endothelial cell injury in the etiology of atherosclerosis. Nihon Rinsho 46: 515-519.

23. Li A, Peng W, Xia X, et al. (2017): Endothelial-to-mesenchymal transition: a potential mechanism for atherosclerosis plaque progression and destabilization. DNA Cell Biol 36: 883-891.

24. Tuttolomondo A, Di Raimondo D, Pecoraro R, et al. (2012): Atherosclerosis as an inflammatory disease. Curr Pharm Des 18: 4266-4288.

25. Terkeltaub R, Boisvert WA, Curtiss LK (1998): Chemokines and atherosclerosis. Curr Opin Lipidol 9: 397-405.

26. Lopez AD, Mathers CD, Ezzati M, et al. (2006): Global and regional burden of disease and risk factors, 2001: systematic analysis of population health data. Lancet 367: 1747-1757.

27. Tang Y, Jin X, Xiang Y, et al. (2015): The lncRNA MALAT1 protects the endothelium against ox-LDL-induced dysfunction via upregulating the expression of the miR-22-3p target genes CXCR2 and AKT. FEBS Lett 589: 3189-3196.

28. Nissan A, Stojadinovic A, Mitrani-Rosenbaum S, et al. (2012): Colon cancer associated transcript-1: a novel RNA expressed in malignant and pre-malignant human tissues. Int J Cancer 130: 1598-1606.

29. Xu X, Ma C, Liu C, et al. (2018): Knockdown of long noncoding RNA XIST alleviates oxidative low-density lipoprotein-mediated endothelial cells injury through modulation of miR-320/NOD2 axis. Biochem Biophys Res Commun 503: 586-592.

30. Li T, Xie J, Shen C, et al. (2016): Upregulation of long noncoding RNA ZEB1-AS1 promotes tumor metastasis and predicts poor prognosis in hepatocellular carcinoma. Oncogene 35: 1575-1584.

31. Su W, Xu M, Chen X, et al. (2017): Long noncoding RNA ZEB1-AS1 epigenetically regulates the expressions of ZEB1 and downstream molecules in prostate cancer. Mol Cancer 16: 142 .

32. de Barrios O, Gyôrffy B, Fernández-Aceńero MJ, et al. (2017): ZEB1-induced tumourigenesis requires senescence inhibition via activation of DKK1/mutant $\mathrm{p} 53 / \mathrm{Mdm} 2 / \mathrm{CtBP}$ and repression of macroH2A1. Gut 66: 666-682.

33. Qin B, Xiao B, Jiang T, et al. (2012): Effects of miR-590-5p on ox-LDL-induced endothelial cells apoptosis and LOX-1 expression. Zhong Nan Da Xue Xue Bao Yi Xue Ban 37: 675-681.

34. Yang L, Gao C (2019): MiR-590 inhibits endothelial cell apoptosis by inactivating the TLR4/NF- $\kappa \mathrm{B}$ pathway in atherosclerosis. Yonsei Med J 60: 298-307.

35. Han X, Han X, Wang Z, et al. (2016): HDAC9 regulates ox-LDL-induced endothelial cell apoptosis by participating in inflammatory reactions. Front Biosci (Landmark Ed) 21: 907-917.

36. Markus HS, Mäkelä KM, Bevan S, et al. (2013): Evidence HDAC9 genetic variant associated with ischemic stroke increases risk via promoting carotid atherosclerosis. Stroke 44: 1220-1225.

37. Cao Q, Rong S, Repa JJ, et al. (2014): Histone deacetylase 9 represses cholesterol efflux and alternatively activated macrophages in atherosclerosis development. Arterioscler Thromb Vasc Biol 34: 1871-1879.

38. Smith JD (2014): New role for histone deacetylase 9 in atherosclerosis and inflammation. Arterioscler Thromb Vasc Biol 34: 1798-1799. 